\section{$\S 28$. Modelling Density Limit Phenomena in Toroidal Helical Plasmas}

Itoh, K., Itoh, S.-I. (RIAM, Kyushu Univ.),

Giannone, L. (Max-Planck-Institute für Plasmaphysik, Garching)

In this article, we discuss the physics of the density limit phenomena based on an analytic model of toroidal helical plasmas. The combination of the transport loss of energy and radiation loss is analyzed and the scaling law for the density limit is derived. The parameter dependence of the critical density is explained. In the case of L-mode confinement, an approximate relation like $n_{c} \sim P^{1 / 2} B^{1 / 2} V^{-1 / 3} q^{-1 / 4}$ is derived ( $P$ : heating power, $B$ : magnetic field, $V$ : plasma volume, $q$ : safety factor).

We start from the simple energy balance equation

$$
\frac{d}{d t} W=-\frac{1}{\tau_{E}} W-P_{r a d}+P
$$

where $W$ is the plasma energy, $P_{\text {rad }}$ is the radiation loss, $P$ is the heating power, and $W / \tau_{E}$ are the other loss processes. We take the model that the radiation loss has a form like

$$
P_{\text {rad }}=n^{2}\left(\frac{n_{I}}{n}\right) \xi T^{-\gamma} V
$$

and the transport loss rate has the form as

$$
\frac{1}{\tau_{E}} W=\frac{3 V}{\tau_{E 0}} n^{1-\beta} T^{1+\alpha}
$$

With the model Eqs.(2) and (3), we have the formula of density limit as

$$
n \leq n_{c}
$$

where the upper bound of the density is explicitly given as

$$
\begin{aligned}
n_{c}= & C^{\prime} V^{-(1+\mu) /(2+\mu-\beta \mu)}\left(\frac{n_{I}}{n}\right)^{-1 /(2+\mu-\beta \mu)} \\
& \times \xi^{-1 /(2+\mu-\beta \mu)} \tau_{E(0)}^{\mu /(2+\mu-\beta \mu)} P^{(1+\mu) /(2+\mu-\beta \mu)}
\end{aligned}
$$

In this expression, the numerical coefficient $C^{\prime}$ is given as $C^{\prime}=C^{-(1+\mu) /(2+\mu-\beta \mu)}$.

Equation (5) shows that various quantities dictate the upper bound of the density.
We here introduce the separation of the critical density into two factors as

$$
n_{c}=f_{c l} \cdot f_{c T}
$$

where

$$
f_{c T} \propto P^{c_{1}} B^{c_{2}} L^{-c_{3}} q^{-c_{4}}
$$

with

$$
\begin{aligned}
& c_{1}=\frac{1+\alpha+\gamma}{2+2 \alpha+(1-\beta) \gamma}, c_{2}=\frac{a_{2} \gamma}{2+2 \alpha+(1-\beta) \gamma}, \\
& c_{3}=\frac{3+3 \alpha+\left(3-a_{3}\right) \gamma}{2+2 \alpha+(1-\beta) \gamma}, c_{4}=\frac{a_{4} \gamma}{2+2 \alpha+(1-\beta) \gamma} .
\end{aligned}
$$

The factor $f_{c l}$ comes from the specification concerning on the impurity ions.

The international stellarator scaling law (ISSL-95) is used as a reference empirical formula :

$$
\tau_{E}^{I S S L} \propto n^{0.51} P^{-0.59} B^{(0.83} L^{2.86} q^{-0.4} .
$$

By use of Eq.(8), Eq.(7) provides an approximate form

$$
n_{c} \sim P^{1 / 2} B^{1 / 2} V^{-1 / 3} q^{-1 / 4}
$$

This result is compared to the experimental observation $n_{c} \propto \sqrt{P B / V}$. The dependence on the heating power and magnetic field is close to the observation; Dependence on the plasma size becomes weaker than $n_{c} \propto V^{-1 / 2}$.

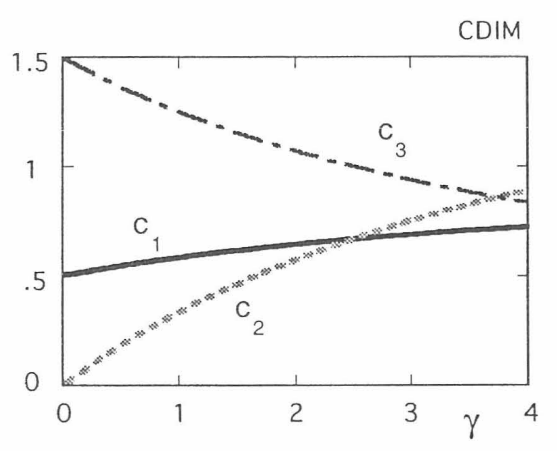

Fig.1 Power index as a function of the gradient of radiation coefficient

Reference

K. Itoh, S.-I. Itoh and L. Giannone: NIFS report $627(2000)$ 